\title{
EFFECT OF VACUUM POLARIZATION IN A STRONG MAGNETIC FIELD AND SPECTRAL FEATURES OF X-RAY SOURCE EMISSION
}

\author{
Yu. N. Gnedin \\ USSR Academy of Sciences Central Astronomical Observatory \\ 196140 Leningrad, Pulkovo, USSR
}

\begin{abstract}
In strong magnetic fields of neutron stars electron-positron vacuum behaves as an anisotropic medium. Vacuum influences the generation and propagation of electromagnetic radiation in plasma and changes the spectrum of radiation. As a result the change of cyclotron lines shape and appearance of specific "vacuum lines" should be observed in X-ray spectra of accreting neutron stars.
\end{abstract}

KEYWORDS. X-ray astronomy; X-ray spectroscopy; pulsars; black holes.

One of the recent remarkable achievements in Astrophysics is the discovery of neutron stars and magnetic white dwarfs which have rather strong magnetic fields $\mathrm{B} \sim 10^{10} \div 10^{9} \mathrm{G}$ and $10^{6} \div 10^{9} \mathrm{G}$, respectively. These magnetic fields are much higher than those achieved in laboratory conditions $\left(10^{6} \div 10^{7} \mathrm{G}\right)$ or those "ordinary" stars $\left(\leq 10^{4} \mathrm{G}\right)$. Such a large difference gives rise to new qualitative effects in the processes involving interaction of radiation with matter (see Pavlov and Gnedin, 1984).

First, they are related to quantization of electron motion transverse to the magnetic field lines. Quantization becomes important if the characteristic energy of electrons appears to be smaller than the distance between Landau levels $\hbar \omega_{\mathrm{B}}=11.6 \mathrm{~B}_{12} \mathrm{keV}$. This condition holds for thermal electrons if

$$
\mathrm{C} \equiv \frac{\hbar \omega_{\mathrm{B}}}{\mathrm{kT}}=1.3 \mathrm{~B}_{12} / \mathrm{T}_{8} \geq 1
$$

Second, the enormous magnetic fields lead also to the appearance of the relativistic quantum effects which are characterized by a rather large ratio:

$$
\hbar \omega_{\mathrm{B}} / \mathrm{m}_{\mathrm{e}} \mathrm{c}^{2} \equiv \mathrm{B} / \mathrm{B}_{\mathrm{c}} \quad \text { where } \mathrm{B}_{\mathrm{c}}=4.41 \times 10^{13} \mathrm{G}
$$

As an example we might present (Fig. 1) the creation of an electron-positron pair (a), the splitting of a photon into two photons (c) and the so-called polarization of electronpositron vacuum (b).

For the case (b) if $\hbar \omega<2 \mathrm{mc}^{2}$ the one-photon creation of real pairs is forbidden by the energy conservation law even in a magnetic field. However the magnetic field affects the virtual electron-positron pairs which are produced due to the photon propagation (Fig. 1b). As a result, the magnetized vacuum behaves with respect to the propagation of photons like an anisotropic medium.

The propagation of radiation in a magnetized vacuum may be described in terms of two normal waves with different polarization and different refractive indices.

$$
\mathrm{n}_{1,2}=1+\frac{1}{90 \pi} \frac{\mathrm{e}^{2}}{\hbar c}\left(\frac{\mathrm{B}}{\mathrm{B}_{\mathrm{c}}} \sin \theta\right)^{2}\left[(7)_{1},(4)_{2}\right]
$$


at $B \ll B_{c}$ and $\hbar \omega \ll m_{e} c^{2}$.

The normal waves are linearly polarized. Under the real physical conditions of the X-ray sources one needs to take into account "vacuum" contribution to the usual plasma effects. It means that the vacuum effects are determined by the ratio of the vacuum polarizability to the plasma polarizability. In the region of the cyclotron resonance $\omega \approx \omega_{\mathrm{B}}$ this ratio is

$$
\frac{\alpha_{\mathrm{v}}}{\alpha_{\mathrm{Pl}}} \equiv \mathrm{V}=\frac{1}{60 \pi^{2} \mathrm{~N}}\left(\frac{\mathrm{m}_{\mathrm{e}} \mathrm{c}}{\hbar}\right)^{3}\left(\frac{\hbar \omega_{\mathrm{B}}}{\mathrm{m}_{\mathrm{e}} \mathrm{c}^{2}}\right)^{4} \cong\left(3 \times 10^{28} / \mathrm{N}\right)\left(\mathrm{B} / \mathrm{B}_{\mathrm{c}}\right)^{4}
$$

where $\mathrm{N}$ is the electron density. For $\omega \ll \omega_{\mathrm{B}}$ the ratio is

$$
\alpha_{\mathrm{V}} / \alpha_{\mathrm{P} 1} \sim \mathrm{V}\left(\omega / \omega_{\mathrm{B}}\right)^{\mathrm{B}_{2}}
$$

and for $\omega » \omega_{\mathrm{B}}$

$$
\alpha_{\mathrm{V}} / \alpha_{\mathrm{P} 1} \sim \mathrm{V}\left(\omega / \omega_{\mathrm{B}}\right)^{4}
$$

The value $\mathrm{V} \sim 1 \div 10^{5}$ follows from estimates of electron densities and magnetic fields in the emission regions of $\mathrm{X}$-ray pulsars: $\mathrm{B} \sim 0.1 \mathrm{~B}_{\mathrm{c}}, \mathrm{N} \sim 10^{19} \div 10^{24} \mathrm{~cm}^{-3}$. Thus, the vacuum polarization in $\mathrm{X}$-ray sources has a strong effect on plasma radiation.

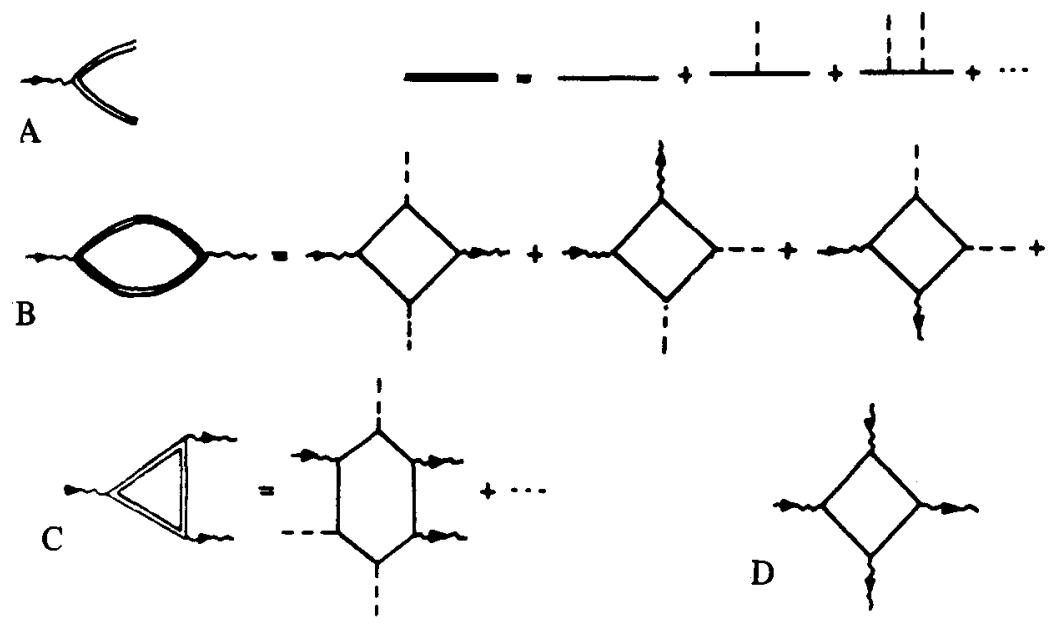

Figure 1. An example of the interaction of photons with the electromagnetic field. (a) Pair production by a photon in a magnetic field; (b) vacuum polarization by a magnetic field (three lower-order diagrams illustrating the interaction of an electron or positron with the magnetic field are shown on the right side); (c) splitting of a photon into two photons (one of the lower-order diagrams illustrating the interaction with the magnetic field is shown on the right side); (d) one of the lower-or ler diagrams for photon-photon scattering. The wavy line represents a photon, the single solid line represents a free electron or a free positron, the double line denotes an electron or a positron in a magnetic field, and the dashed line represents a magnetic field "quantum". 
Though the vacuum phenomena affect mainly the polarization of radiation nonetheless the X-ray line positions and shapes are also determined by vacuum effects, especially for the optically thick plasma. The most pronounced effects arise in the range of wavelengths where the contribution from the vacuum is compensated by that from the plasma. There are frequencies $\omega_{\mathrm{c} 1,2}$ at which the effects of the vacuum and plasma on the linear polarizaion of normal waves cancel out each other: for $V \gg 1$

$$
\omega_{\mathrm{c} 1} \approx \omega_{\mathrm{B}}\left(1-\frac{1}{2 \mathrm{~V}}\right) ; \omega_{\mathrm{c} 2} \approx \omega_{\mathrm{B}} / \sqrt{\mathrm{V}}
$$

These frequencies are close to the points of intersection of dispersion curves for the absorption coefficients and refraction indices (see Fig. 2).

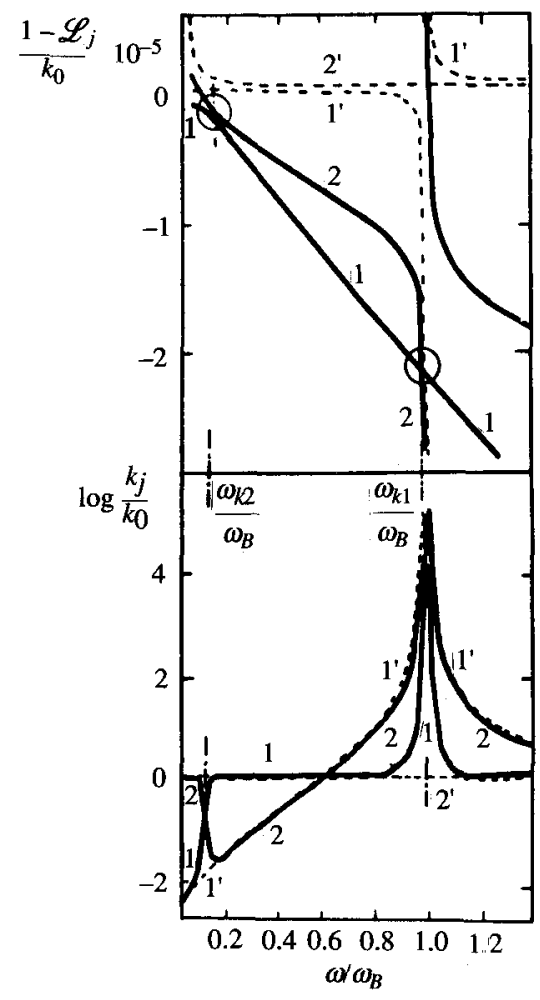

Figure 2. Frequency dependence of the refractive indices and absorption coefficients of normal waves for $\mathrm{V}=100, \theta=85^{\circ}$. The numbers 1 and 2 near the curves denote the number of the wave $j$ ( 1 ' and 2' for the "vacuumless" plasma). $K_{0}=(c / 2 \omega) N \sigma_{T}=K_{d}$ ' $B=$ 0 ). The regions of enhanced linear transformation of normal waves in an inhomogeneous plasma are circled.

Although dth $\omega \ll \mathrm{m}_{\mathrm{e}} \mathrm{c}^{2}$ the vacuum cannot absorb the photons directly it has nonetheless a strong effect on the absorption of radiation by plasma. This results from the fact that the absorption of radiation is an anisotropic medium depends essentially on its polarization which is changed by the vacuum (Fig. 3 ).

The main result due to the vacuum effect is the increasing of the contribution from second normal wave near cyclotron resonance. In the usual thermal plasma cyclotron 
resonance absorption is practically determined by the first normal wave: $K_{2} \sim \beta K_{1}$, where $\beta=\left(2 \mathrm{kT} / \mathrm{mc}^{2}\right)^{1 / 2}$. In the vacuum case $(\mathrm{V} » 1)$ the cyclotron absorption coefficients are to be of the same order where $K_{2} \sim K_{1}$ (Fig. 4). This striking difference from the vacuumless plasma is due to the change in the selection rules for the absorption of normal waves.

As a result the behaviour of absorption coefficients $\mathrm{K}_{\mathrm{j}}$ near the first cyclotron resonance $\omega_{\mathrm{B}}$ turns out to be rather complex (Fig. 5). The profiles of $\mathrm{K}_{\mathrm{j}}(\omega)$ acquire a complex shape, additional points $\omega_{\mathrm{K}}$ at which $\mathrm{K}_{\mathrm{j}}$ or refraction indices $\mathrm{n}_{\mathrm{j}}$ crossing appear, additional shifts and dips occur as a result of vacuum polarization.

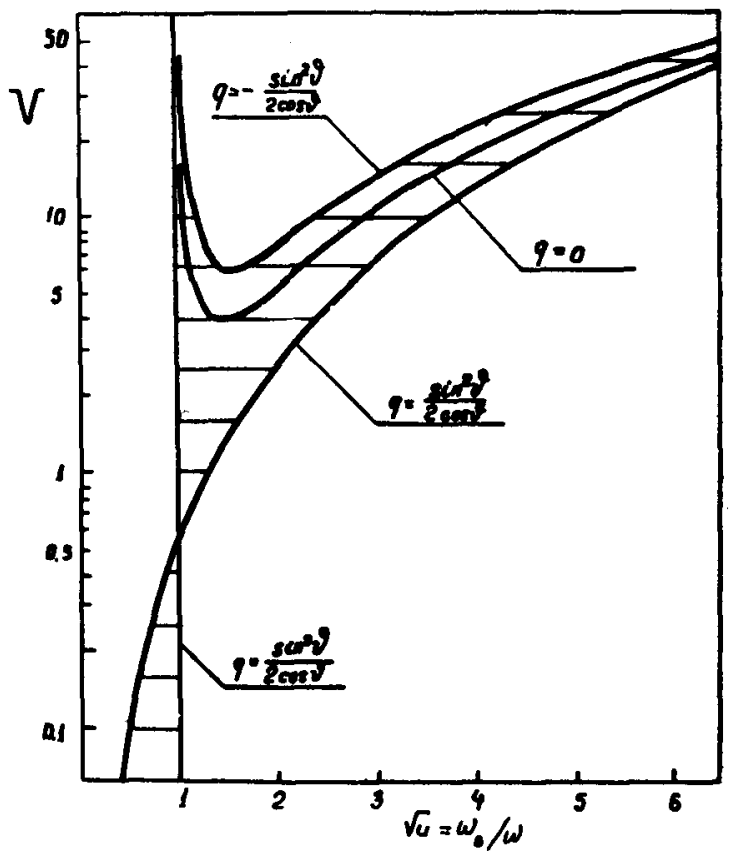

Figure 3. The regions of quasi-transverse and quasi-longitudinal (the hatched area) propagation in the "plasma + vacuum" system. The vacuum polarization changes considerably the polarization of normal waves above the curve $\mathrm{q}=\sin ^{2} \theta / 2 \cos \theta$.

The quantizing field $(b \geq 1)$ causes the cyclotron resonance to shift by $S^{2} \omega_{B}\left(B / 2 B_{c}\right) \sin ^{2} \theta$ and the quantity

$$
\xi=\mathrm{S} \frac{\mathrm{B}}{\mathrm{B}_{\mathrm{c}}} \tanh \frac{\mathrm{C}}{2} \sin ^{2} \theta
$$

which takes into account the contribution of M1 transitions with spin flip to increase. Furthermore, as the magnetic field is increased at a fixed temperature, the absorption at a higher-order harmonics increases $\sim \mathrm{B}^{\mathrm{S}-1} / \mathrm{S}$ at $\mathrm{b} \gg 1$ due to the change of the thermal energy $k T$ of the transverse motion of electrons by the quantum energy of Landau levels $\hbar \omega_{\mathrm{B}}$ and due to the decrease in the role of the induced transitions. This situation is valid only for not too high harmonics $S \ll B_{d} / B$ when the profiles do not overlap each other. As 

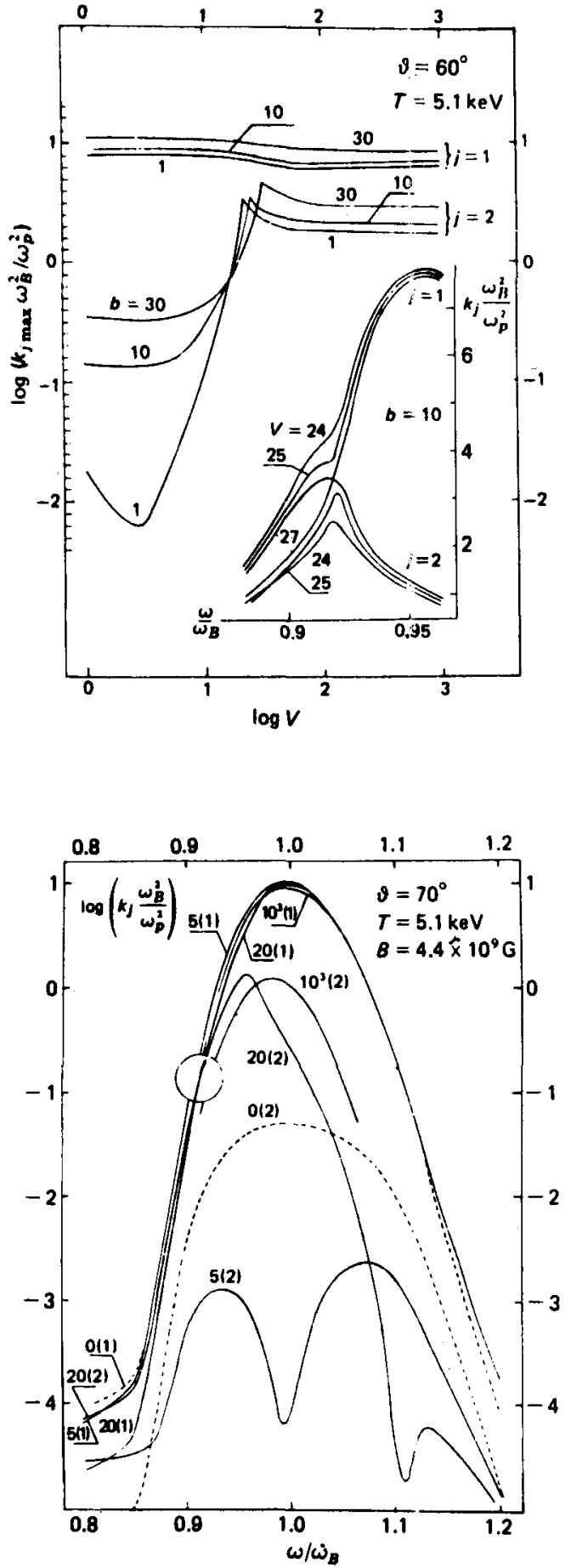

Figure 4. Top: Dependence of the maxima of the absorption coefficients of the normal waves neart the main resonance on the paramater $V$ for $\theta=60^{\circ}, \beta=0.141$ and different values of $b$ indicated on the curves. The maximum values of $\mathrm{K}_{2}$ are attained at $\mathrm{V}=\mathrm{V}_{0}$. The curves $K_{j} \max (V)$ intersect at lower values of $\theta$ Bottom-right: A change in the profiles of $k_{j}(\omega)$ occurring when $\mathrm{V}$ passes through $\mathrm{V}_{0}=25.2$ at $\mathrm{b}=10$.

Figure 5. The effect of vacuum polarization on the absorption of normal waves near the first resonance at $\beta=0.141$ and $\theta=70^{\circ}$ for $b$ « 1 . The circle indicates the point of intersection of the $\mathrm{k}_{\mathrm{j}}$ curves for $\mathrm{V}=20$. 
$\mathrm{b}$ is increased the resonances overlap each other at lower $\mathrm{S}$ due to quantum shift.

The vacuum polarization has no effect on the intensity of radiation of optically thin plasma, since the sum $k_{1}+k_{2}$ is independent of $V$. Therefore the vacuum changes the frequency and angular dependences of the polarization characteristic of radiation. In contrast with this case the vacuum changes both the polarization properties and the intensity of optically thick plasma radiation because the intensity $I=I_{1}+I_{2}$ depends nonlinearly on absorption coefficients $K_{j}$.

For example, the peaks caused by the vacuum polarization at $\omega \approx \omega_{\mathrm{B}}(1-2 / \mathrm{V})$ and at $\omega \approx \omega_{\mathrm{B}} \mathrm{V}^{-1 / 2}$ in the frequency dependence of the smaller absorption coefficient in a cold plasma lead to additional absorption lines in the spectrum of the emitted radiation (Fig. 6 ). One of these lines appears near the cyclotron frequency $\omega_{\mathrm{B}}$. In a cold plasma without scattering it arises only due to vacuum polarization, although both thermal motion and the conversion of normal waves during scattering give rise to the cyclotron line without allowance for the vacuum effect. The other line at $\omega \approx \omega_{\mathrm{B}} \mathrm{V}^{-1 / 2}$ whose position depends on the plasma density appears exclusively because of vacuum polarization.

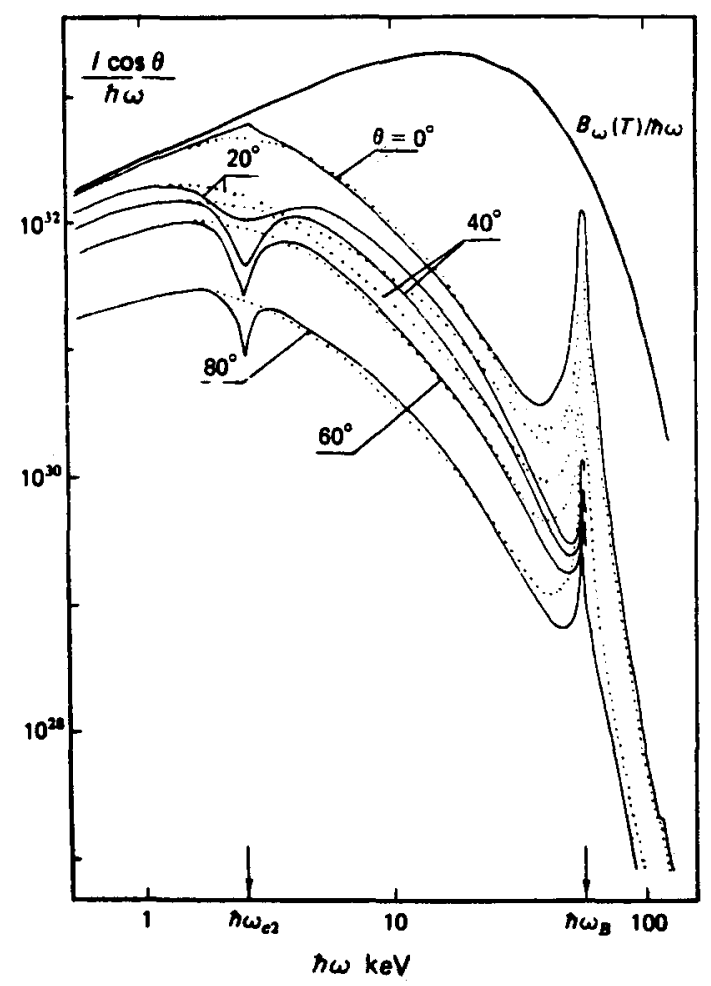

Figure 6. Spectra of partial fluxes from a semi-infinite plasma calculated with and without allowance for vacuum polarization $(\mathrm{V}=480$ and $\mathrm{V}=0$ solid and dashed curves, respectively) for $\mathrm{kT}=10 \mathrm{keV}, \mathrm{B}=5 \times 10^{12} \mathrm{G}, \mathrm{N}=10^{22} \mathrm{~cm}^{-3}$ and different angles $\theta$ between the normal and wave vector. Magnetic field is perpendicular to the surface. 

obtain

For a simple commonly accepted model of X-ray pulsar (Lamb et al., 1973) one can

$$
\begin{gathered}
\mathrm{V}(\mathrm{r})=150 \frac{\mathrm{B}_{12}^{24 / 7} \mathrm{R}_{6}^{67 / 7}}{\mathrm{~L}_{37}^{5 / 7} \mathrm{r}_{6}^{19 / 2}}\left(\mathrm{M} / \mathrm{M}_{\odot}\right)^{19 / 4} \\
\hbar \omega_{\mathrm{c} 2} \cong 0.88 \mathrm{~L}_{37}^{5 / 14} \mathrm{r}_{6}^{19 / 4} \mathrm{M}_{\odot}^{19 / 28} / \mathrm{B}_{12}^{5 / 7} \mathrm{R}_{6}^{67 / 14} \mathrm{M}^{19 / 28} \mathrm{keV}
\end{gathered}
$$

For Her X-1 the value $\hbar \omega_{\mathrm{c} 2} \sim 0.1 \mathrm{keV}$. For $4 \mathrm{U} 0115+634$, if the feature near $20 \mathrm{keV}$ is assumed to be a cyclotron absorption line, the value $\hbar \omega_{c 2} \approx 0.55 \mathrm{keV}$. In other models these values may be larger. For example, it may be suggested that the absorption features in spectrae of $\gamma$-ray bursts discovered by Mazetz et al. (1981) can be possibly explained as the vacuum features. One can suppose that the peculiar emission near $12 \mathrm{keV}$ observed in the spectrum Her X-1 by McCray et al. (1982) is a vacuum feature near $\omega_{\mathrm{c} 2}$. Thus we would have $\mathrm{V} \approx 3400$ in the emission region. It gives the electron density value $\mathrm{N}=3 \times$ $10^{21} \mathrm{~cm}^{-3}$ for $\mathrm{B}=6 \times 10^{12} \mathrm{G}$. It should be noted that a simultaneous detection of vacuum and cyclotron features make it possible to uniquely determine the magnetic field and the electron density in the emission region.

\section{REFERENCES}

Lamb, F.K. , Pethick, C.J. and Pines, D. (1973), Astrophys. J., 184, 271.

Mazets, E.P., Golenetskii, S.V., Aptekar', R.L., Gur'yan, Yu.A. and Il'inskii, V.I. (1981), Nature, 290, 378.

McCray, R. A., Shull, J.M., Boyton, P.E., Deeter, J.E., Holt, S.S. and White, N.E. (1982), Astrophys. J., 262, 301.

Pavlov, G.G. and Gnedin, Yu.N. (1984), Astrophys. Space Physics, v. 3, 197-253. 